\title{
Astrophysical point source search with the ANTARES neutrino telescope
}

\author{
S. Mangano for the ANTARES Collaboration
}

IFIC - Instituto de Física Corpuscular, Edificio Institutos de Investigación, Apartado de Correos 22085, 46071, Spain

\begin{abstract}
The ANTARES neutrino telescope is installed at a depth of $2.5 \mathrm{~km}$ of the Mediterranean Sea and consists of a three-dimensional array of 885 photomultipliers arranged on twelve detector lines. The prime objective is to detect high-energy neutrinos from extraterrestrial origin. Relativistic muons emerging from charged-current muon neutrino interactions in the detector surroundings produce a cone of Cerenkov light which allows the reconstruction of the original neutrino direction. The collaboration has implemented different methods to search for neutrino point sources in the data collected since 2007. Results obtained with these methods as well as the sensitivity of the telescope are presented.
\end{abstract}

Keywords: Neutrino telescope, point source search, clustering analysis, upper limits, neutrino flux

\section{Algorithms to search point sources}

The following two unbinned clustering algorithms are used to search for neutrino point sources (signal) in the sky map of atmospheric neutrinos (background). The Expectation-Maximization algorithm is based on an analytically likelihood maximization [1]. The signal density distribution is assumed to be a two-dimensional gaussian and the background density distribution is taken from the data. The probability to have a signal for a given background model is maximized. The free parameters are the two sigmas of the two-dimensional gaussian probability density function and the expected number of signal events from the source.

The Likelihood algorithm calculates first the angular distance between a fixed source search point and the location of all selected neutrinos in the sky. Then it fits this distribution with signal and background density distribution using maximization technique. The signal distribution is the point spread function taken from Monte Carlo and the background distribution is taken from data.

\section{Data results}

A set of cuts have been optimized to search for an $E^{-2}$ neutrino flux in the data sample taken in 2007 with a livetime of 140 days. An all sky point source search based on the above algorithms has not revealed any significant excess for any direction. No significant excess has been found also in a dedicated search from a selected list of 24 promising neutrino source candidates. The upper limits on the neutrino flux from the 24 candidate sources are shown in Figure 1

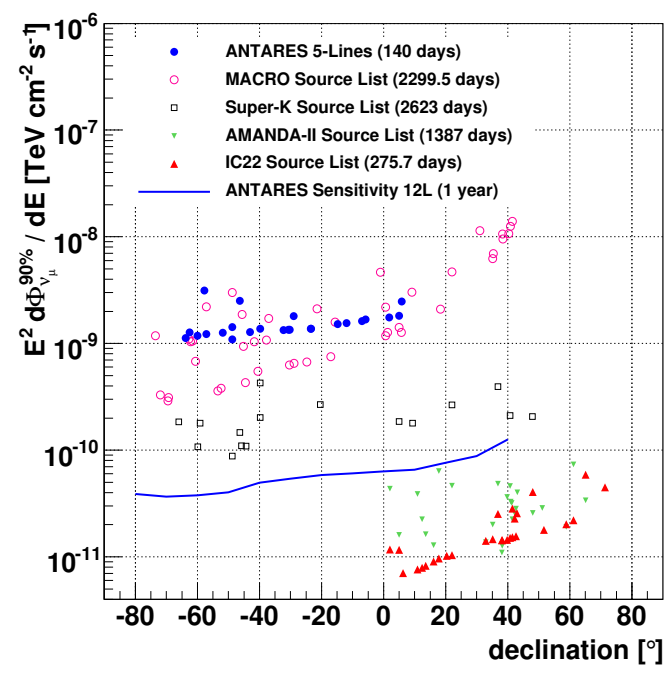

Figure 1: The upper limits from the 24 candidate sources for 2007 data (filled points) compared with the results published by other neutrino experiments (Macro [2], Super-K [3], Amanda [4] and IceCube [5]). The predicted sensitivity of ANTARES for 365 days (line) is also shown.

\section{References}

[1] J. Aguilar and J. Hernandez-Rey, Astrop. Phys. 292 (2008) 117.

[2] M. Ambrosio et al, Astrophys. J. 546 (2001) 1038.

[3] S. Desai et al, Astrop. Phys. 29 (2008) 42.

[4] R. Abbasi et al, Astrophys. J. Lett. 701 (2009) 47.

[5] R. Abbasi et al, Phys. Rev. D 79 (2009) 062001.

\section{Acknowledgments}

I gratefully acknowledge the support of the JAE-Doc postdoctoral programme of CSIC. This work has also been supported by the following Spanish projects: FPA2009-13983-C02-01, MultiDark Consolider CSD2009-00064, ACI2009-1020 of MICINN and Prometeo/2009/026 of Generalitat Valenciana. 